\title{
Method Of Defogging Image Based On the Sky Area Separation
}

\author{
Yanhai $\mathrm{Wu}^{1, a}$, Kang ${ }^{1}$ Chen, Jing ${ }^{1}$ Zhang, Lihua Pang ${ }^{1}$ \\ ${ }^{1}$ College of Communication and Information Engineering, Xi'an University of Science and \\ Technology, Xi'an, 710054, China \\ aEmail:wyh7388@163.com,
}

\begin{abstract}
Keywords: Image Defogging, Image Enhancement, Sky Separation, Adaptive Histogram Equalization.
\end{abstract}

\begin{abstract}
Under bad weather, the outdoor computer vision system captures the image quality degradation, which will bring great inconvenience to image processing, has a serious impact on aspects of daily life and production. In this paper, From the point of image enhancement, first, the image is converted from the RGB color range to HSV color range of the color image, only the luminance computer is separated from the sky region, the non-sky area carries out adaptive histogram equalization processing, the hue and saturation component remained unchanged. The experiment results demonstrate that the algorithm can effectively improve image degradation and improve the image's contrast under foggy.
\end{abstract}

\section{Introduction}

In foggy, the most significant feature of the atmosphere is significantly reduced visibility. In such weather conditions, lots of the important messages in image are covered, which have different levels for all walks of life. Such as during heavy fog UAV aerial survey will seriously affect the management and supervision of department workers, Criminal image data plays an increasingly important role in Criminal Case of Public Security. If outdoor surveillance systems to encounter inclement weather conditions, it will brings great difficulties for police officers to extract useful image information. Therefore, a series disadvantages caused by the low image contrast, high brightness, the research is particularly important in how defogging.

Currently, Fog image clarity is mainly used in atmospheric scattering physical model-based approach targeted, defogging effect obtained naturally, which will be no loss of information, but this method requires many assumptions and estimates a lot of parameters in [1]. For example, a way, which is based on the physical model to restore the contrast of the scene and the scene depth, which need to measure the calibrated Radar. is proposed in [2]. Using polarization of the light to get two photographs of the same scene under different weather conditions, the ambient light is estimated by calculating the difference of the light in different polarization direction, then restore the image, this method requires to estimate too many parameters in [3] [5]. Global histogram equalization algorithm in [4] [6] is image enhancement in a common method, which is simple, practical and suitable for image depth slowly changing images. Local histogram equalization in [7] [8] greatly improves the contrast-enhanced image of local information in each local region, but this method will be enhanced gray area with slow varying by mistake(such as sky region), thereby leading to the restoration pictures affected by noise unnatural. The algorithm in document [8] proposes the color image in the RGB color space is respectively sky area division in the three channels. But color images in three color channels more closely linked, and the segmenting threshold calculated and compared the size repeatedly, which brings to increase the time consumption.

In view of the shortcomings of the above algorithm, In this article puts forward a foggy image conversion from the RGB color space to the HSV color space, only the luminance component is extracted, selecting the appropriate threshold separation sky region and the non-sky area, the sky region maintains its brightness unchanged, non-sky area adaptive histogram equalization, the results will be restored to the RGB color space. 


\section{The separation of the sky and non-sky area}

According to the formation of fog, mist with a strong light scattering and reflection, the image's foggy area of the sky gray value is relatively high. Although the entire region of the gray sky is not a constant, gray values changes in a limited range of variation. In the fog, the luminance histogram in HSV color image, the larger value has a steep peak, the interval in which the peak is located corresponds to the range of sky luminance variation area (see in Fig.1 (b)). In one image, the depth of the sky region considered to be infinity, it can be approximately the same depth. Therefore the distribution of its gray values should satisfy the normal distribution. Given the fog and image luminance histogram show in Fig. 1.

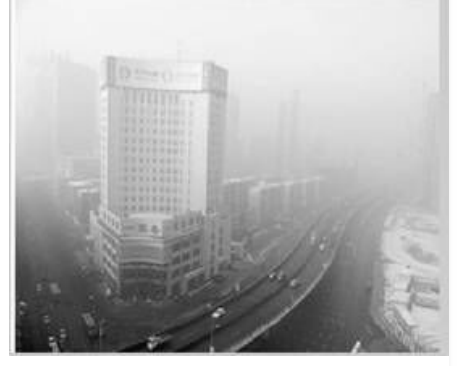

(a) The fog

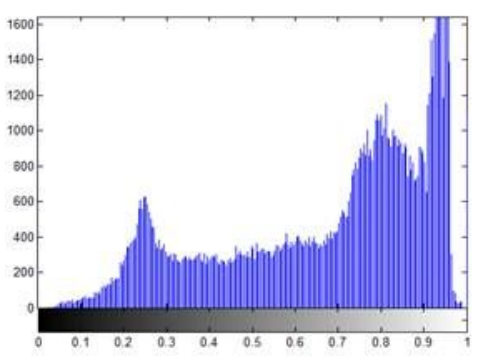

(b) luminance histogram

Fig.1 Fog and luminance histogram

\subsection{Best approximate normal search algorithm}

Image brightness distribution curve and approximate normal distribution curve shown in Fig. 2. First, luminance component curve scanned from the right to left (solid blue line in Fig 2), the highest peak was referred to as $\left(h_{\max }, p_{\max }\right)$, the horizontal axis of the point (the image brightness level) as the initial mean luminance $\mu$ of the normal distribution (et: $h_{\max }=\mu$ ), according to the given standard deviation $\sigma$, then a normal distribution can be obtained, such as the Eq. 1.

$$
y=p_{\max } e^{\frac{(h-\mu)^{2}}{2 \sigma^{2}}}
$$

Eq. 1 is a one-dimensional Gaussian function, among $p_{\max }$ is the Amplitude, $\mu$ represents the mean luminance of Gaussian function, $\sigma$ represents the standard difference of the Gaussian function. The deviation of normal distribution and brightness distribution curve is:

$$
\text { eps }=\sum_{h=0}^{255}|y(h, \sigma)-p(h)|
$$

In Eq.2, $h$ indicates the luminance level of the luminance component. $y(h, \sigma)$ represents the Gaussian function value on the corresponding brightness level of h. $p(h)$ corresponds to the frequency of the h luminance level. eps represents the deviation between the Gaussian function and the luminance curve.

By searching for satisfying the Eq.3, the indicator $\hat{\sigma}$ is the best approximation of the variance of the normal distribution. The Eq. 3 as follows:

$$
J(\widehat{\sigma})=\min \left\{\sum_{h=0}^{255}|y(h, \sigma)-p(h)|\right\}
$$

Specific Steps are as follows:

(1)Given an initial variance $\sigma_{0}$, we can receive the initial normal distribution through substituting $\sigma_{0}$ into the Eq.1, also calculated initial deviation $e p s_{0}$

(2) Set $\sigma=\sigma_{0}+\delta_{\sigma}$ into the Eq.1, it was used to calculate the new normal distribution y and the deviation eps . 
(3) Judging from whether the initial deviation $e p s_{0}$ is greater than $e p s$, if it is true, then go to step 2, re-enter an appropriate value $\sigma$ to calculate the eps. Or it is false, the variance $\sigma$ and the mean value $\mu$ of the normal distribution is the best approximation of normality distribution (red dashed line in Fig. 2).

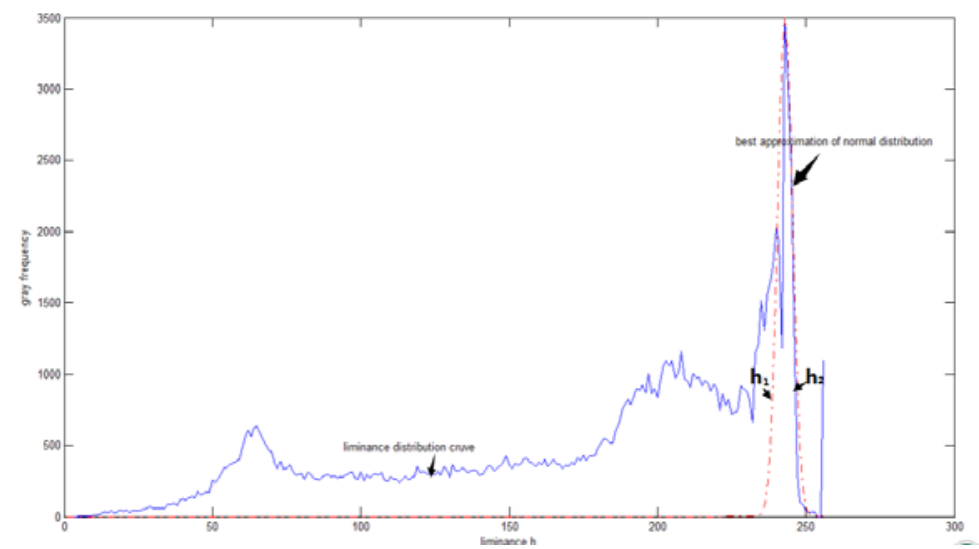

Fig.2 Luminance distribution curve and the best approximation of normality distribution

\subsection{The determination of the sky threshold segmentation}

According to the nature of the normal distribution, when $\mu-2 \sigma \leq h \leq \mu+2 \sigma$ (et. the luminance value is within this range $\left[h_{1}, h_{2}\right]$ ) wherein $h_{1}=\mu-2 \sigma, h_{2}=u+2 \sigma$ is the range of brightness distribution of sky area (shown in Fig. 2), the probability distribution is about 95 percentage of the total distribution. If a gray scale image, this method can be used directly as the gray distribution range of sky area. For color images, the image is converted to HSV color space, the separation of the sky area is based on the interval of the range $\left[h_{1}, h_{2}\right]$ in luminance component, and the image processing in luminance component doesn't affect the saturation and hue component. According to the separate range of $\left[h_{1}, h_{2}\right]$, the segmentation results for housing photos sky region is shown in Fig.3

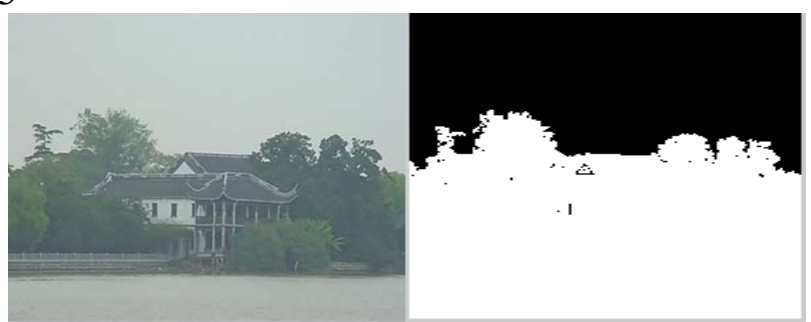

(a) Housing photo (b) Segmentation results

Fig. 3 House photos sky region segmentation map

\section{Defogging treatment of the image}

When the gradation transformation slow region was enhanced, the algorithm in article [4] has a shortcoming that the sky area will be enhanced by mistake. Literature [8] proposed to solve this error is to find out the sky area of the foggy, and then the local histogram equalization is performed on the non-sky area after the separation of the sky region. As can be seen from the house fog photos, as long as the contrast of the image to make appropriate increase, it can achieve the purpose of clarity. Since the great differences in different depths of the object shown in the fog, the algorithm in literature [8] proposed to non-sky area selection of a suitable size of mobile template, and template scenes can be approximately regarded as the same depth, then the scene with same depth in the template block-overlapping histogram equalization processing. This paper proposes to use an adaptive histogram equalization (AHE) for the non-sky region, first, the local block of the image distribution is processed by histogram equalization, and then the bilinear interpolation is used to splice the pieces together in order to eliminate the gray level difference caused by the local block.

The size of the image is $\mathrm{M}^{*} \mathrm{~N}$, output image is $e 1(x, y)$, here are specific steps: 
(1)Initialize two variables $x, y$, and give the initial number $x=1, y=1$; and initialize three same size of $\mathrm{M}^{*} \mathrm{~N}$ zero matrixes $g, e 0, e 1$, wherein $g$ is the output image area of the sky, $e 0$ is the non-sky area, $e 1$ is the output of the defogging image;

(2)According to the luminance histogram of the image, using the sky area segmentation method mentioned before extracts to the best approximation to meet the optimal normal range $\left[h_{1}, h_{2}\right]$, which is used to segment the sky region.

(3) The current point $(x, y)$ is determined whether this point belongs to the area of the sky, if it is true, then $g(x, y)=f(x, y)$; or if it is false, then go to the next step;

(4) Mark all the point of the sky area, the non-sky region of the segmented image $e 0$ is obtained by adaptive histogram equalization;

(5)Copying the $f(x, y)$ to the corresponding to the output image $g(x, y)$;

(6)The output image el consists of the sky area and the non-sky area.

\section{The algorithm processes}

In Literature [8], the algorithm is the original color image directly in the RGB color space to the separation of area of the sky, the enhanced image changed the color of original color image. This paper through the transformation of color space, only the image brightness component processing, area of sky to maintain the same brightness, adaptive histogram equalization processing non sky area to increase the image contrast. The experimental results in the subjective more in line with the human visual characteristics. The specific flow chart is shown in Figure 4.

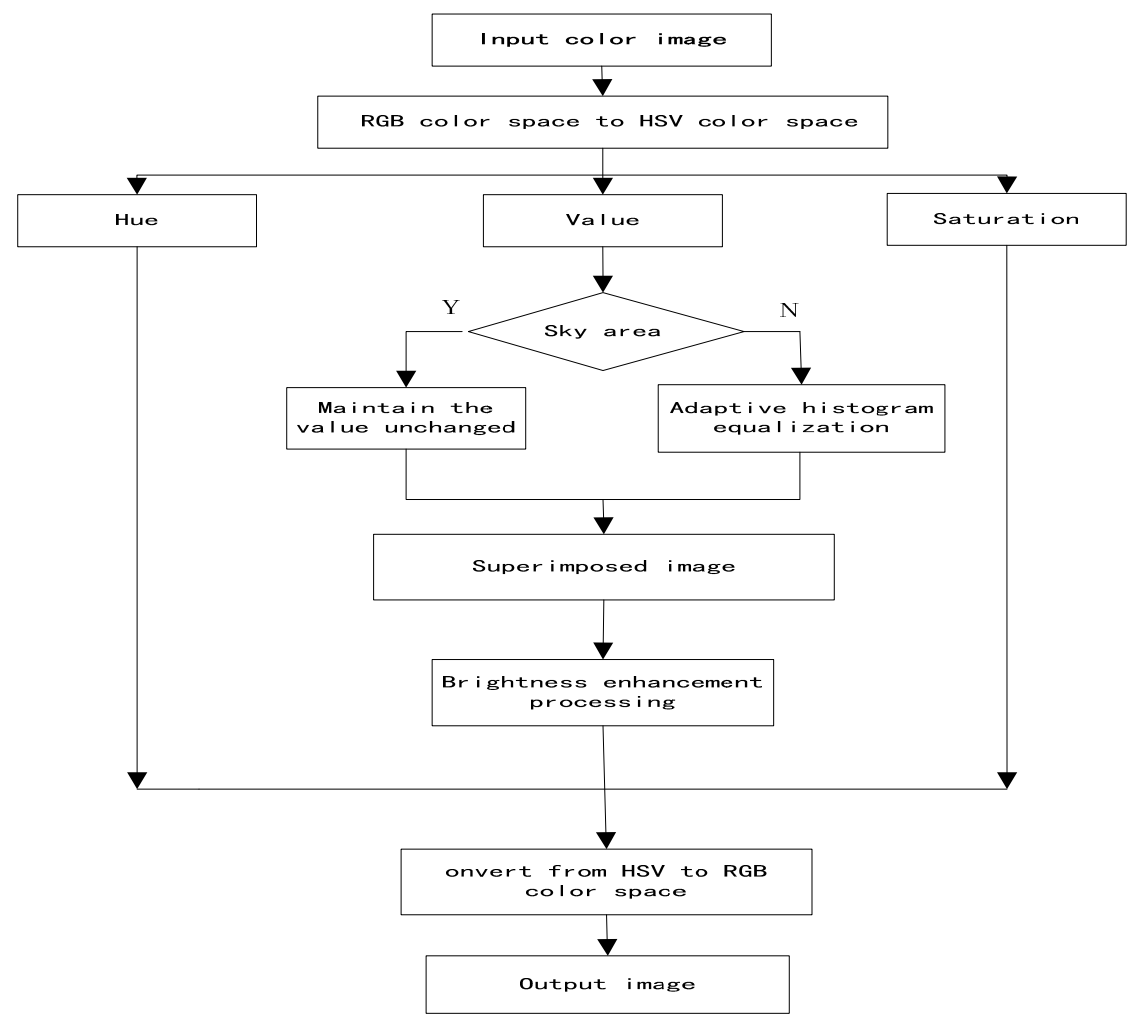

Fig. 4 Algorithm flow chart

\section{Test results}

In this paper, the experiment using computer Inter(R) Core(TM) i3-2330 CPU@2.2 GHz, memory is $4 \mathrm{G}$, the operating system is Windows 7, experimental platform for MATLAB R2013b. 
The proposed algorithm will be compared to the results of the literature [8] algorithm defogging effects as shown in Fig. 5.
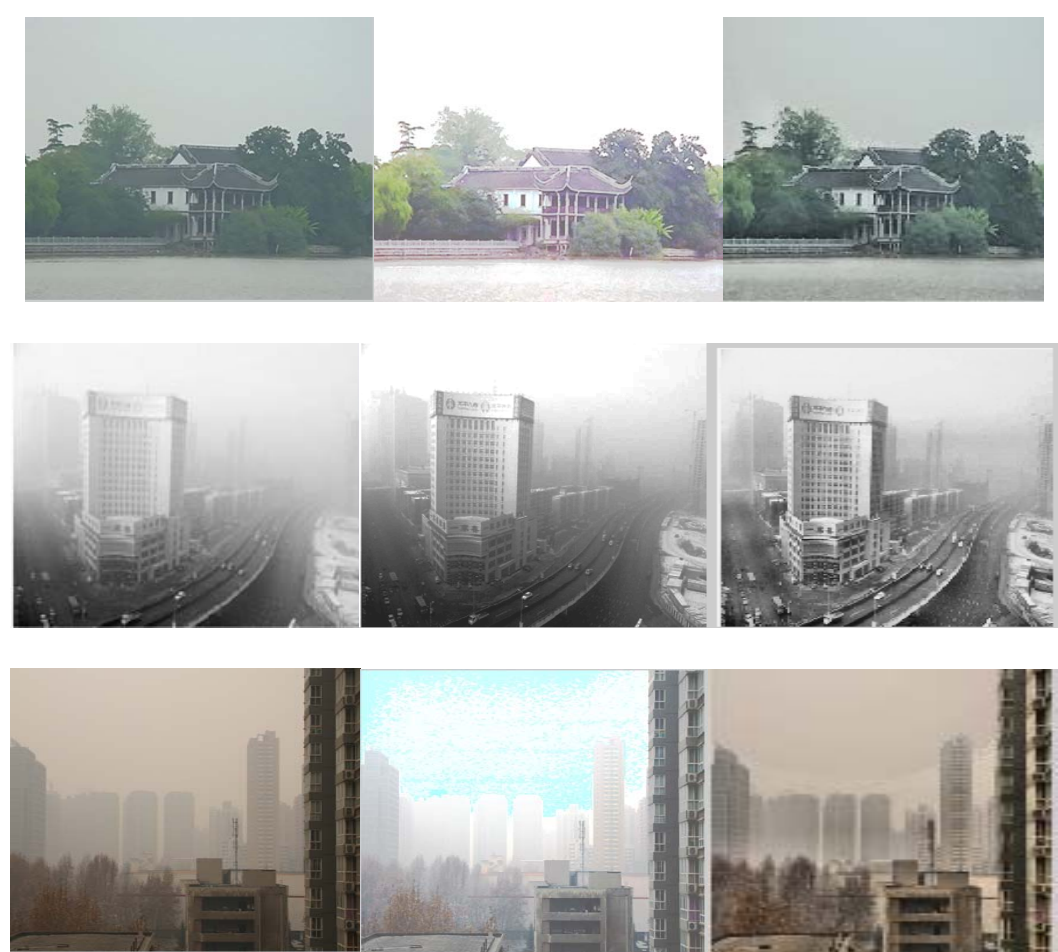

Original image Literature [8] result the algorithm result

Fig. 5 the experimental results

From Fig. 5, the result of [8] in the second group is dark, the image's outline of the details don't recognize, but in this paper, the result of the image's brightness is more brighter and more in line with the subjective feelings of the human eye; The first and third groups of [8] processing results have been significant distorted in the sky area, the result of this paper in keeping with the original color is more consistent, more carefully, especially you can see the outline of the leaf in the first group. In the second group can be seen the far three chimney shape, and shape of the window in the distant buildings legible in the third group. This algorithm to enhance the fog image is more realistic.

Table1 Defogging objective image quality evaluation of FIG. 5

\begin{tabular}{cccccc}
\hline $\begin{array}{c}\text { Image } \\
\text { name }\end{array}$ & $\begin{array}{c}\text { Algorithm } \\
\text { type }\end{array}$ & $\begin{array}{c}\text { Average } \\
\text { gradient }\end{array}$ & Entropy & $\begin{array}{c}\text { Standard } \\
\text { deviation }\end{array}$ & time \\
\hline \multirow{2}{*}{ Group 1 } & Article[8] & 4.9566 & 4.8061 & 11.9243 & 0.6058 \\
& Paper Alg. & 6.3855 & 6.4591 & 11.9429 & 0.5379 \\
Group 2 & Article[8] & 4.2313 & 5.4610 & 10.9372 & 0.9791 \\
& Paper Alg. & 8.8256 & 7.0984 & 11.1018 & 0.5365 \\
Group 3 & Article[8] & 5.1141 & 6.5705 & 11.1173 & 1.5802 \\
& Paper Alg. & 7.0469 & 7.0164 & 11.6266 & 1.4423 \\
\hline
\end{tabular}

This article select the average gradient, entropy and the standard deviation of the image as an objective evaluation to verify the validity of the algorithm, from an objective evaluation of the parameters in Table 1 can be seen that the information entropy and average gradient in this paper than the literature [8] height is about 1.4 times, the speed of the algorithm faster than literature [8] is about 1.2 times. Standard deviation, are improved but only slightly.

\section{Conclusion}

In order to highlight the color image enhancement, this paper presents an image based on image enhancement algorithms to fog. This paper selects the individual components independent HSV 
color model, and only the sky area separated in luminance component, hue and saturation components remains unchanged, non-sky area adaptive histogram equalization processing, and the defogging image obtained either in color or brightness of the above are more consistent with human visual perception. Experimental results show that the proposed method can not only effectively improve the degradation and close-range vision and contrast of the image, but also the enhanced image illumination uniformity, details clearly.

\section{Acknowledgement}

In this paper, the research was sponsored by the National Natural Science Foundation of Shaanxi Province (Grant No. 2015JQ6221). Scientific and Technological Research Project of Science and Technology Department of Shaanxi Province (Grant No. 2012K06-16).

\section{References}

[1] GUO Fan, CAI Zi-xing, XIE Bin, TANG Jin ET all. Review and prospect of image dehazing techniques [J]. Journal of Computer Applications, Vol.30, No.9, 2010: 2417-2421(In China);

[2] Oakley John, P. Satherley Brenda L. Improving image quality in poor visibility conditions using a physical model for contrast degardation [J]. IEEE Transactions on Image Processing, 1998, 7(2):167-179;

[3] WANG Song, XUE Mu-gen, HUANG Qin-chao, Polarization Dehazing Algorithm Based on Atmosphere Background Suppression[J]. Computer Engineering, Vol.35,No.4,2009:271-272(In China);

[4]Kim Tae Keung, Paik Joon Ki, Kang Bong Soon. Contrast enhancement system using spatially adaptive histogram equalization with the temporal filtering [J]. IEEE Transactions on Consumer Electronics, 1998,44(4): 82-86;

[5]Shwartz S. Namer S, Schechner YY. Blind haze Separation[C]// Proceedings of IEEE Conference on Computer Vision and Pattern Recognition (CVPR). New York,NY.USA::IEEE computer Society,2006.2:1984-1991;

[6]J Frankle. J McCann. Method and Apparatus for Lightness Imaging. [J].US Patent 1983.5(4):1738-1743;

[7] Joung-Youn Kim, Lee-Sup Kim, Seung-Ho Hwang An Advanced Contrast Enhancement Using Partially Overlapped Sub-Block Histogram Equalization. IEEE on Circuits and System for video Technology, Vol.11, No.4, 2001:475-484;

[8] ZHU Pei, ZHU Hong, QIAN Xue-ming,et all. An Image Clearness Method for Fog[J]. Journal of Image and Graphics, Vol.9, No.1, Jan. 2004: 124-128(In China); 